\title{
Editorial Comments to the Special Issue of Neuropsychology Review on the Basic Neuroscience and Neuropsychology of Selective Movement Disorders
}

\author{
Tilman Schulte $^{1,2} \cdot$ Helen Brontë-Stewart $^{3}$
}

Received: 17 November 2015 / Accepted: 17 November 2015/Published online: 21 November 2015

(C) Springer Science+Business Media New York 2015

Neuropsychology aims to understand the relations among mind, brain, and behavior. Everyday activities such as walking, talking, and writing involve motor skills that are intertwined with selective cognitive abilities. The complexity of this interaction is reflected in the neuroanatomy of the motor system that is characterized by dissociable basal gangliacortical circuits for planning, selection, initiation, execution, and self-monitoring of behavior. To achieve a better understanding of similarities of motor and cognitive deficits in patients with damage to specific basal ganglia-cortical circuits, this special issue on the basic neuroscience and neuropsychology of selective movement disorders features reviews on Parkinson's disease (PD) and other disorders with Parkinson-like symptoms.

PD is a well-studied neurodegenerative disorder that affects as many as $1-2 \%$ of people $\geq 60$ years showing motor abnormalities that include signs of involuntary movement (hyperkinetic), intentional motor activity (hypokinetic), or both. Even before motor symptoms are evident, non-motor symptoms can emerge, such as depression, constipation, compromised olfaction, and sleep disorders, that can adversely affect functioning in daily life. As the disease progresses, the loss of dopaminergic neurons in the substantia nigra pars compacta leads to striatal dopaminergic depletion, generating both motor

Tilman Schulte

tilman.schulte@sri.com

1 Neuroscience Program, Bioscience Division, SRI International, Menlo Park, CA, USA

2 Pacific Graduate School of Psychology, Palo Alto University, Palo Alto, CA, USA

3 Department of Neurology \& Neurological Sciences, Stanford University School of Medicine, Stanford, CA, USA symptoms and executive-function deficits and other behavioral alterations. For a mechanistic and clinical account of possible cognitive impairments in motor disorders, a systematic neuropsychological evaluation of motor and non-motor cognitive abilities is necessary for identification of which specific basal ganglia-cortical motor system is implicated. The reviews in this issue of Neuropsychology Review on the Basic Neuroscience and Neuropsychology of Selective Movement Disorders provide perspectives on the underlying mechanisms of movement disorders and give insight into new clinical approaches that target motor and cognitive impairment at different stages of PD.

The topics review basic neuroscience on neurotransmitter pathways and mechanisms with new discoveries on the role of nicotinic receptor-mediated dopaminergic signaling that may offer a novel therapeutic approach with nicotinic receptor drugs as neuroprotective agents to prevent PD progression (Perez). Further reviewed are established therapies for the treatment of patients with PD, such as high frequency (HF) deep brain stimulation (DBS) of the subthalamic nucleus (STN) that effectively reduces the disabling motor symptoms including tremor, bradykinesia, and rigidity (Blumenfeld \& Brontë-Stewart). The chronically implanted DBS electrodes also enable the characterization of underlying neural rhythms from several nodes in the PD network to explore the relationship between brain and behavior, both acutely and over time, with clinical implications for targeting specific motor symptoms. DBS further led to a mechanistic understanding of the role of the STN in non-motor limbic functions pertaining to impulse control and valence processing with implication for treating impulse control disorders in PD overall and specifically in patients on dopamine agonist therapy (Rossi \& Okun). In addition, the development of protein biomarkers to identify PD patients at risk for cognitive impairments for effective treatment is discussed (Leaver \& Poston). Recent studies 
provided evidence that low baseline $A \beta 42$ in the cerebrospinal fluid (CSF) of non-demented PD patients predicts development of cognitive impairment over time. Parkinson-like symptoms have also been observed in people living with HIV infection (DeVaughn, Müller-Oehring, Markey, BrontëStewart, \& Schulte). With the introduction of highly active anti-retroviral therapy (HAART), individuals diagnosed with HIV are living longer. Extended longevity raised a query whether aging HIV-1 infected individuals are at heightened risk for developing neurodegenerative conditions similar to PD with damage to a basal ganglia-cortical circuitry affecting motor and cognitive functions. Recognition of similarities in subcortico-cortical connectivity and patterns of cognitive and motor impairment in aging HIV and PD, relative to normal aging, should advance identification of potential neural targets for treatment. Finally, a meta-analysis sheds light on the effec- tiveness of DBS targeting the STN or the globus pallidus interna (GPi) for improving cognitive, behavioral, and mood symptoms in addition to improving motor symptoms and dyskinesias (Combs, Folle, Berry, Segerstrom, Han, AndersonMooney, Walls, \& van Horne). Interestingly, stimulating STN and GPi are equally effective at improving motor symptoms and dyskinesias; however, unclear is whether it affects cognitive, behavioral, and mood symptoms differently.

The contributions of this special issue are aimed at advancing our awareness and understanding of recent scientific developments spanning basic neuroscience to the clinical treatment of selective movement disorders while acknowledging the complexity in the interaction between motor symptoms and cognitive functioning in patients with damage to specific basal ganglia-cortical circuits. 